\title{
An electrifying selection of virtual reading
}

\section{Compiled by Helen Nield}

This issue's selection is made up entirely of ebooks. If you have not tried an ebook please email library@bda.org or call 02075634545 for access details. Members can read these books online at any time or download most titles for up to a week before having to download them again. Visit bda.org/ebooks to find all of these books and more.

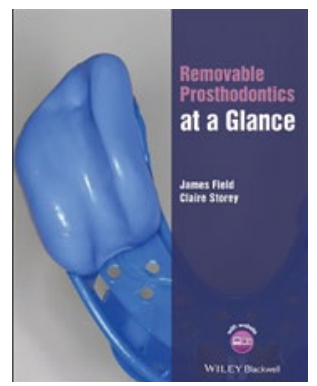

\section{REMOVABLE PROSTHODONTICS AT A GLANCE}

James Field and Claire Storey

2020; Wiley Blackwell; 120 pp; ebook

ISBN: 9781119510741

'Providing prostheses that are

satisfactory to the patient is a challenge and there are many reasons why patients can be dissatisfied with the finished result. Many relate to social aspects of patients' lives ... there is often a common thread running through [the diversity of complaints] - lack of information exchange and an inappropriate level of patient expectation. We would therefore argue that the most important skill when making satisfactory removable prostheses is that of communication.'

This new addition to Wiley's 'At a Glance' series covers the difficult topic of removable dentures both complete and partial. It covers the basics in short easy to read chapters full of simple diagrams and easily digestible text along with an accompanying website with quiz questions and downloadable assessment forms. The book consists of 47 short chapters looking at everything from the function of removable prostheses to positioning of the dentist at different stages of prosthetic treatment and all aspects of production, techniques and problems. The appendices consist of proformas for complete denture assessment and restorative assessment, referral letters, a partial denture design sheet and a reading list. An excellent book that can be used both as an introduction to the subject and as a revision guide. https://tinyurl.com/ ebooksglance

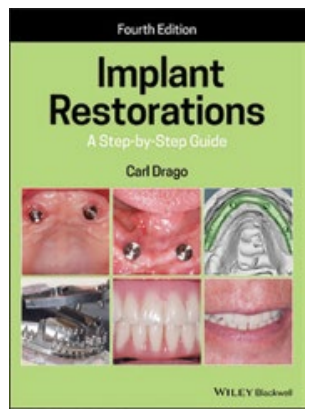

IMPLANT RESTORATIONS: A STEP-BYSTEP GUIDE (4TH EDITION)

Carl Drago

2019; Wiley-Blackwell; 536 pp; ebook

ISBN: 9781119538110

'The purpose of this textbook is to provide clinicians and dental laboratory technicians with a step-by-step approach to the treatment of certain types of edentulous and partially edentulous patients with dental implants. Six types of patient treatments with multiple implant loading protocols, have been featured.'

This new edition of a well-known textbook has been fully updated and expanded since it was last published in 2014. New to this edition are digital dentistry techniques that have become available in the last few years. The text is full of images including CT scans and pictures from software programs as well as photographs and diagrams. Each chapter begins with a literature review and ends with a bibliography and a fully comprehensive clinical case presentation. Treatments that are covered are, 'Treatment of edentulous mandibular patients,' 'Replacement of single teeth with CAD/CAM implant restorations', 'Fixed dental prostheses: retreatment of a patient with a fractured implant-retained fixed dental prosthesis', 'Accelerated treatment protocol of a patient with edentulous jaws and CAD/CAM titanium framework/fixed hybrid prostheses', 'Treatment of edentulous patients with immediate occlusal loading: conventional surgical and computer-generated surgical guides, 'Treatment of edentulous patients with immediate occlusal loading: conventional surgical/prosthetic protocols, 'Treatment of partially edentulous patients with immediate non-occlusal loading protocols. The book finishes with a chapter covering guidelines and maintenance procedures for fixed, full-arch, implantretained prostheses. https://tinyurl.com/ ebookimplants

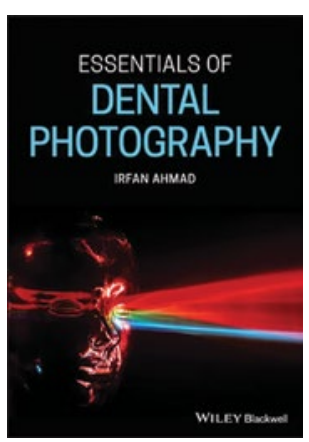

\section{ESSENTIALS OF DENTAL PHOTOGRAPHY}

Irfan Ahmad;

2019; Wiley-Blackwell; 360 pp; ebook ISBN: 9781119312147

'[M]any clinicians are reticent about incorporating dental photography into their daily practice due to uncertainty about the choice of equipment, a steep learning curve and initial capital expenditure. These fallacious notions are fuelled by the plethora of dental literature on the subject, some scientifically based, some anecdotal, while others perversely complicate what is basically a simple procedure. It is the endeavour of this book to demystify many of these erroneous beliefs by proposing protocols for standardising photographs that are invaluable for intra- and inter-patient comparison.' 
4 This title is a very practical text written by the author of previous books and articles on the subject including a digital photography series of articles that appeared in the BDJ in 2009. This new book covers the basics of photography that a dental practitioner would need to start taking their own images. It is split into three sections: Equipment and concepts; Photographic set-ups; and Processing images. The book looks not only at intraand extra-oral imaging but also covers the taking of full head portraits, 'bench images' which incorporate macrophotography and imaginative shots for promotional and teaching purposes and some special advanced applications such as 'taking additional images for elucidating certain features, or analysing images for effective communicating with patients and fellow colleagues.' The book finishes with a look at how images can be used both in terms of clinical practice documentation and for marketing purposes. https://tinyurl.com/ ebookphotography

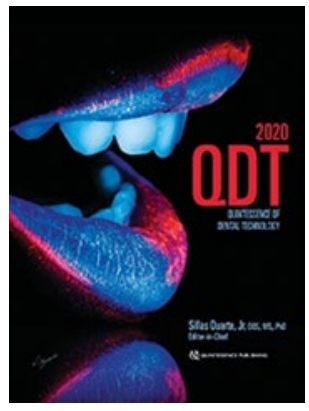

\section{QUINTESSENCE OF DENTAL} TECHNOLOGY (QDT) VOLUME 43, 2020

Silas Duarte, Jr (Editor)

2020; Quintessence Publishing Co; 257 pp; ebook ISBN: 9781647240141

'Digital dentistry and facially generated treatment planning (smile design) are closely related. Natural reconstructions with application of digital facial scanning and analysis as well as other digital technologies vital to all phases of clinical dentistry are required in current esthetic restorative treatment.' - from the article Digital minimally invasive esthetic treatment.
This annual publication comprises new articles on various aspects of dental technology by a variety of different authors. This latest offering contains articles by, amongst others, Mario Alessio Allegri, James Choi, Douglas Terry, Cristiano Soares and Naoki Hayashi. A bumper edition, it includes a short editorial on computational photography and 17 different articles. Minimally invasive dentistry is represented by articles on 'The pillars of full-mouth rehabilitation: a minimally-invasive, low-cost approach to prosthetic treatment' and 'Digital minimally invasive esthetic treatment'. Amongst other topics there are pieces on subjects as diverse as digital workflow, self-glazing liquid ceramics and photopolymerisation. If you can ignore the many pages of adverts between the articles then this is a great book to browse through to pick up ideas on current technology and marvel at the art of the possible. https://tinyurl.com/ ebooktechnology

\section{Public back calls to extend sugar tax}

Data collected by the Oral Health Foundation as part of National Smile Month shows that $61 \%$ of the United Kingdom support an expansion of the current Soft Drinks Industry Levy - also known as the sugar tax.

Milkshakes, fruit juices, smoothies and alcoholic mixers, which are exempt under the current sugar tax, all received equal backing as possible routes for an extension.

A previous report looking into some of the drinks exempt from the sugar tax found that half contain a child's entire recommended daily sugar intake, which is almost $19 \mathrm{~g}$ or nearly five teaspoons.

Dr Nigel Carter OBE, Chief Executive of the Oral Health Foundation, believes the Soft Drinks Industry Levy has had a positive impact on the nation's health and supports calls to extend the sugar tax further.

Dr Carter said: 'The sugar tax has been a significant success, not only for oral health, but for general health and wellbeing too.

The more sugar we can continue to cut from

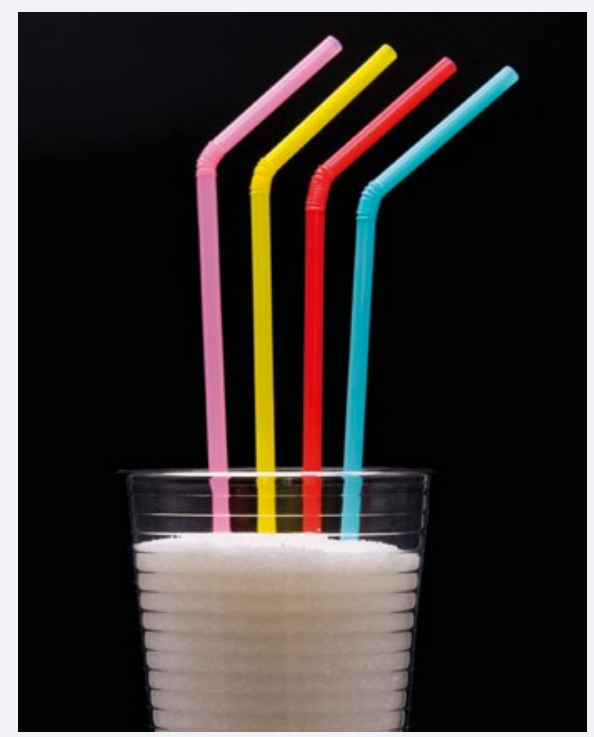

drinks, the healthier our population will be. It will allow more of us to be free of the diseases and conditions linked to sugar, and it will also save the NHS millions every year.

'The lack of progress by government to build on the current sugar tax proposals has been extremely disappointing. Expanding the sugar tax to include milkshakes, smoothies and fruit juices is a relatively small step but the impact it could have would be enormous.'

The sugar tax was introduced two years ago and applies to drinks with more than $8 \mathrm{~g}$ of added sugar per $100 \mathrm{ml}$. The tax forced manufacturers to lower their sugar content or face a tax rate equivalent to $24 \mathrm{p}$ per litre. As a result, many of them did. So much so that the new levy brought $£ 800$ million less than it was forecast to.

Since then, the sugar content of drinks sold has fallen by $21.6 \%$ - equating to more than 30,000 tonnes of sugar a year.

'The impact that sugar has on teeth is horrific,' said Dr Carter. 'It is why one-inthree adults in the UK have tooth decay and it is the reason why around 35,000 children are admitted to hospital each year.

During National Smile Month, the Oral Health Foundation is challenging the public to cut its added sugar intake. Advice on sugar swaps is available at www. smilemonth.org. 